\title{
POSITRON IRRADIATION EFFECTS ON POSITRONIUM FORMATION IN POLYCARBONATES DURING A POSITRON-ANNIHILATION EXPERIMENT
}

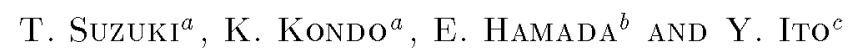 \\ ${ }^{a}$ High Energy Accelerator Research Organization (KEK) \\ Tsukuba, Ibaraki 305-0801, Japan \\ ${ }^{b}$ Department of Radiological Sciences, Ibaraki Prefectural University of Health Sciences \\ Ami, Ibaraki 300-0394, Japan \\ ${ }^{c}$ Research Center for Nuclear Science and Technology, The University of Tokyo \\ Tokai, Ibaraki 319-1106, Japan
}

The positron-irradiation effect on polycarbonate during a positronannihilation lifetime spectroscopy experiment was investigated using different intensities of ${ }^{22} \mathrm{Na}$ positron sources and $\gamma$-irradiated samples. The decrease in $I_{3}$ was larger for a larger intensity of positron sources. In the case of a weak source $(\approx 140 \mathrm{kBq}), I_{3}$ did not change with the elapsed time for non-irradiated samples. However, for $1 \mathrm{MGy}$ irradiated samples, $I_{3}$ measured with the weak source increased with the elapsed time. This can be attributed to a decrease in the radicals induced in the irradiated samples by $\gamma$-irradiation. In order to explain the change in $I_{3}$ measured at room and liquid-nitrogen temperatures, several effects, such as radicals, cross-linking, structure change, and charging, need to be considered. Also, it is difficult to explain the change in $I_{3}$ using only one of these effects.

PACS numbers: $78.70 . \mathrm{Bj}$

\section{Introduction}

The positron-irradiation effect has been discussed and increases and decreases in the positronium (Ps) formation have been reported at low temperature [1-4] and room temperature [5-7], respectively. Before observing Ps quenching by irradiating polymer samples with visible light $[8-10]$, the increase at low temperature was explained by the relaxation behavior of polymer structures. A quenching experiment has demonstrated that Ps formation is accelerated by an increase in the trapped electrons, which are induced by the ionization of injected 
positrons, which are trapped in a shallow potential being formed among polymer structures at low temperature [8-10]. This is a phenomenon similar to the well-known "anti-inhibition or anti-recombination effect" [11].

The decrease in $I_{3}$ at room temperature has been explained by many ideas, such as cross-links [12], structure change [13], and radicals [14]. A structure change and the cross-links were introduced to explain the decrease in $I_{3}$ at low $\gamma$-irradiation doses of around $10 \mathrm{kGy}$, since, at a low irradiation dose, it seems to be difficult to expect radicals which affect the change of $I_{3}$.

In this studies, the reduction of Ps formation has been explained using several effects, mentioned above, and the effect of positive charges, which constantly accumulate by injecting positrons in polymers from positron sources during PALS measurements.

\section{Experiment}

The PALS experiments were conducted using a conventional fast-fast coincidence system having a time resolution of 270-300 ps at full width at half maximum (FWHM). The details of the apparatus have been presented elsewhere [7].

The polycarbonate (PC) sample used in the experiment was commercially available from Sigma-Aldrich Japan K.K.; the sample was supplied in pellet form and was melted at $320^{\circ} \mathrm{C}$ to form a plate of $1-2 \mathrm{~mm}$ thickness. The plate was then cut into pieces of $10 \mathrm{~mm} \times 210 \mathrm{~mm}$ for PALS experiments.

Positron sources were prepared by depositing and drying aqueous ${ }^{22} \mathrm{NaCl}$ on a $7 \mu \mathrm{m}$ thick kapton film having an area of $10 \mathrm{~mm} \times 10 \mathrm{~mm}$, which was then covered with the same size of film. In order to investigate the dependence on the positron source, three kinds of sources with intensities of $3.7(100), 0.96(26)$, and $0.18(5) \mathrm{MBq}(\mu \mathrm{Ci})$ were employed.

In order to study the effect of $\gamma$-ray irradiation, the PC samples were sealed in glass tubes under a vacuum and irradiated at room temperature at the ${ }^{60} \mathrm{Co} \gamma$-ray irradiation facility, Japan Atomic Energy Research Institute (JAERI). After keeping the samples at room temperature for more than one week, they were used for PALS experiments, and the free radicals induced by the irradiation were measured by ESR (JEOL JES-RE3X). Hence, this work was concerned with long-lifetime free radicals.

The PALS spectra were automatically saved every one or two hours, resulting in 1 to 2 million events in each spectrum. All spectra were resolved into three components using the PATFIT program [15]; the longest lifetime component (intensity $I_{3}$, lifetime $\tau_{3}$ ) was related to ortho-Ps (o-Ps).

\section{Results and discussion}

Polycarbonate has aromatic rings in the structure, which can absorb the energy of radiation, and the $G$ value of scissions is known to be 10 to 50 times smaller than those of polyethylene $[16,17]$. Hence, ionized electrons may be produced less efficiently than polyethylene by radiation. Generally, a PALS experiment is conducted by sandwiching the positron source between two polymer samples, which 
are continuously irradiated by the probe positrons, whose energy is distributed from 0 to $540 \mathrm{keV}$ and the average energy is about $220 \mathrm{keV}$. From the irradiation, the maximum energy deposit was estimated to be about $400 \mathrm{~Gy} / \mathrm{h}$ for a $3.7 \mathrm{MBq}$ source in a small part located within $20 \mu \mathrm{m}$ from the surface [18]; the accumulated dose within $1 \mathrm{~h}$ was sufficient to produce $10^{16}-10^{17}$ radicals $/(\mathrm{g} \cdot \mathrm{h})$. Also, during the PALS experiments, positron probes were constantly adding positive charges to polymer samples, resulting in the accumulation of charge. Thus, radicals and positive charges were accumulating with the elapsed time of the PALS measurement.

Figure 1 shows the normalized $I_{3}$ versus the elapsed time during PALS experiments using three kinds of intensities of ${ }^{22} \mathrm{Na}$ sources for the non-irradiated

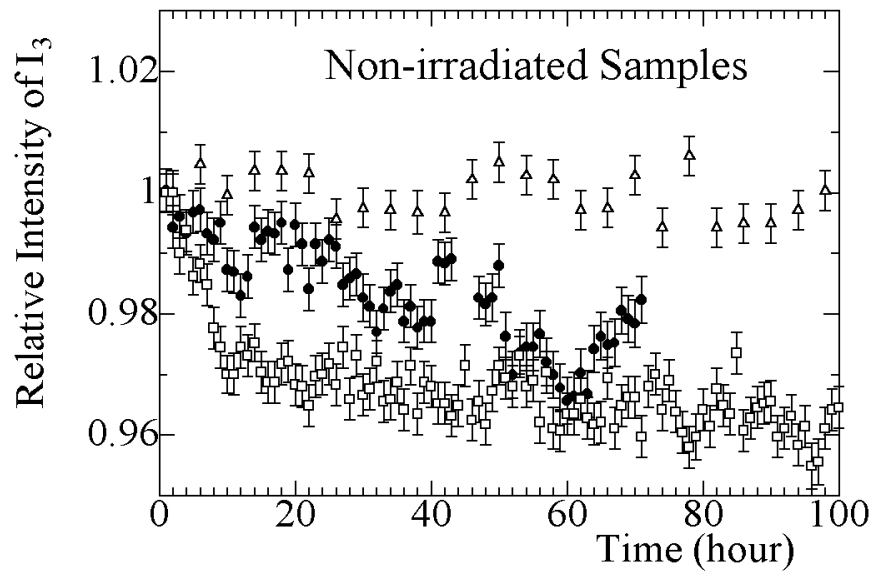

Fig. 1. Normalized $I_{3}$ versus the elapsed time for the non-irradiated PC samples during PALS experiments using three kinds of intensities of ${ }^{22} \mathrm{Na}$ sources: open triangle $0.18 \mathrm{MBq}(5 \mu \mathrm{Ci})$; solid circle $0.96 \mathrm{MBq}(26 \mu \mathrm{Ci})$; open square $3.7 \mathrm{MBq}(100 \mu \mathrm{Ci})$.

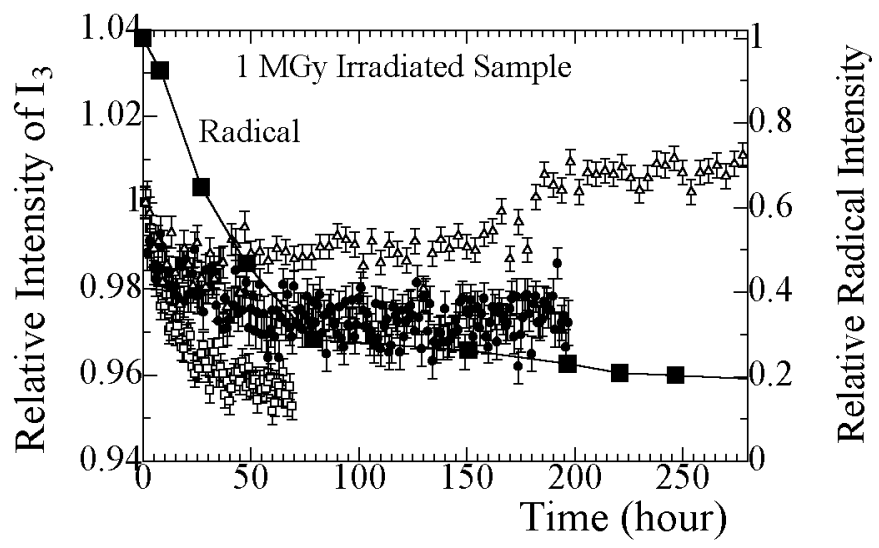

Fig. 2. Normalized $I_{3}$ and radicals (solid square) versus the elapsed time for $1 \mathrm{MGy}$ irradiated PC samples; symbols are the same as listed in the caption of Fig. 1. 
samples. In this experiment the positron sources were always sandwiched by two polymer samples, and the time of the $x$-axis shows both the elapsed time of the measurement and the irradiation time. It indicates that the decreasing rate of $I_{3}$ depends on the intensity of the source, and that no decrease is observed for the $0.18 \mathrm{MBq}$ source (Fig. 1 shows up to $100 \mathrm{~h}$ ). The same measurements were conducted using $1 \mathrm{MGy}$ irradiated samples at the ${ }^{60} \mathrm{Co} \gamma$-ray facility (JAERI); the decreasing rate also depends on the intensity of the source (Fig. 2). However, in the case of a weak source $(0.18 \mathrm{MBq}), I_{3}$ seems to increase after $150 \mathrm{~h}$ of elapsed time. Figure 2 shows the relative radical intensities versus the elapsed time. Since, after $150 \mathrm{~h}$, the radical intensity decreased to $20 \%$ of the initial value, the increase in $I_{3}$ after $150 \mathrm{~h}$ is considered to be attributed to a decrease in the radical density.

Figures 3 and 4 show $I_{3}$ versus the elapsed time measured using strong $(3.7 \mathrm{MBq})$ and weak sources $(0.18 \mathrm{MBq})$, respectively. $I_{3}$ of the non-irradiated sample was larger than that of 1 MGy irradiated samples by $3-4 \%$. This may
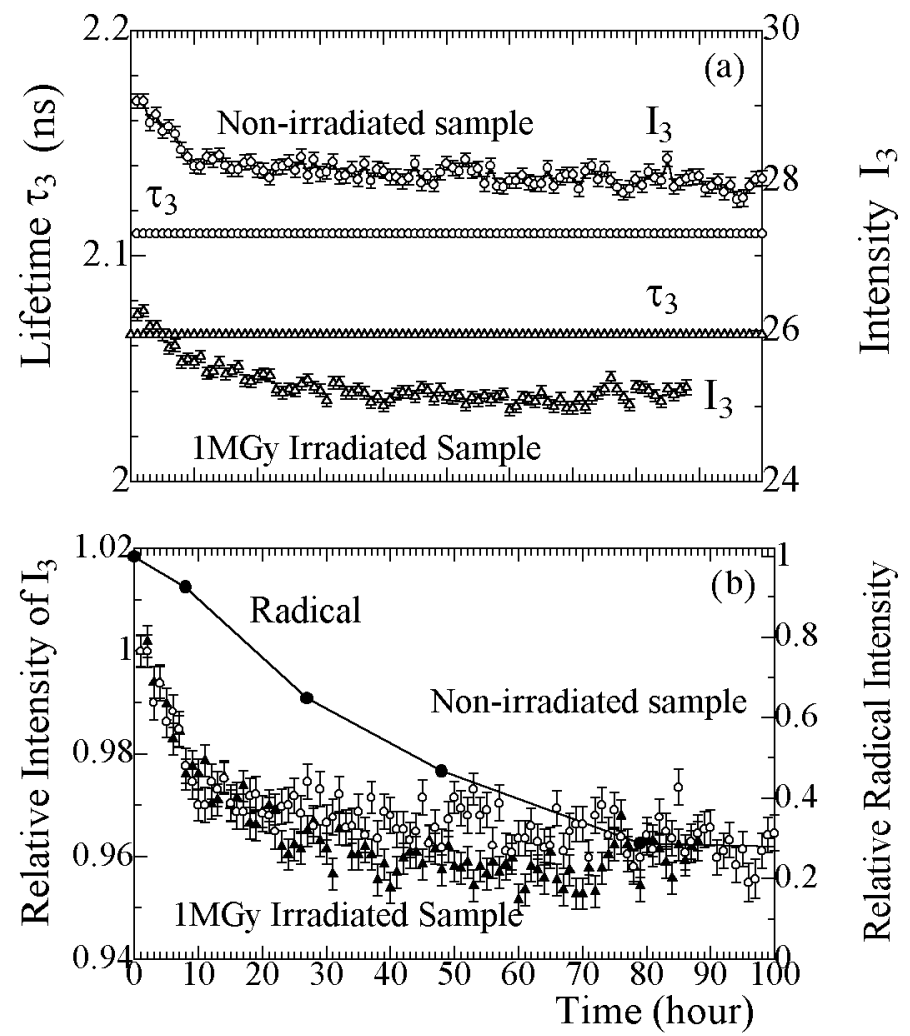

Fig. 3. (a) Lifetimes and intensities versus the elapsed time for the irradiated and non-irradiated PC samples using the strong source of 3.7 MBq during PALS experiments. (b) Normalized intensities and radicals for the data shown in part (a). 


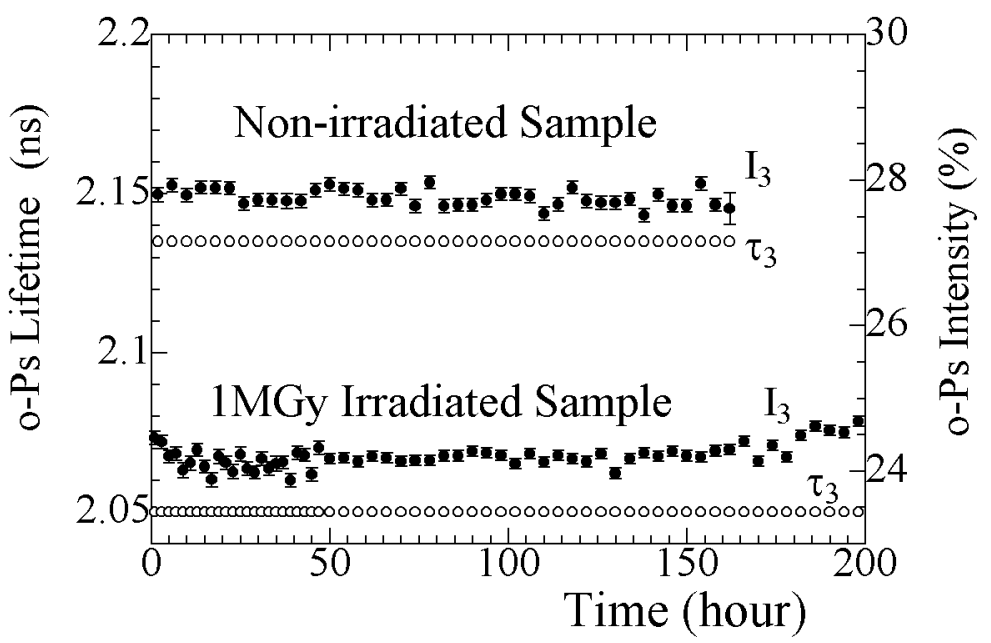

Fig. 4. Lifetimes and intensities versus the elapsed time for the irradiated and non-irradiated PC samples using the weak source of $0.18 \mathrm{MBq}$ during PALS experiments.

be attributed to the radicals, since, in the 1 MGy irradiated sample, radicals of $3 \times 10^{18} / \mathrm{g}$ were observed even after more than one week. Also, $\tau_{3}$ of the irradiated samples was smaller than that of the non-irradiated samples. This indicates that a structure change due to cross-linking occurred, and that the intermolecular spaces shrunk. Thus, the decrease in both $I_{3}$ and $\tau_{3}$ can be attributed to radicals and cross-linking, which are generally expected to be induced in irradiated samples.

In the case of the strong source, the decreasing rate of $I_{3}$ for the $1 \mathrm{MGy}$ irradiated samples was almost the same for the non-irradiated samples. If the cause of the decrease was due to radicals, the decreasing rate in the irradiated samples may not be the same as that of non-irradiated samples, since the number of radicals induced by the source at the beginning of the PALS experiment is smaller than the number of radicals existing before the measurement. Hence, the decrease in $I_{3}$ may not have been caused by radicals, and may be attributed to the accumulation of charges. After a 1 hour PALS measurement, the accumulation was estimated to form an electric field larger than $30 \mathrm{kV} / \mathrm{cm}$, which is sufficient to affect Ps formation [11]. For a weak source, because $I_{3}$ does not change for non-irradiated samples, the effects of radicals and charges, which are induced by the positron source, may be small.

At low temperature, $I_{3}$ showed different variation from that at room temperature. For non-irradiated samples, Fig. 5 shows that $I_{3}$ began to increase as soon as the sample was cooled down to liquid nitrogen temperature. However, for the irradiated samples, $I_{3}$ decreased at the beginning, and then started to increase after $200-220 \mathrm{~h}$. The increase in $I_{3}$ of non-irradiated samples can be explained by trapped electrons [8-10], which are trapped in the potentials created after the freezing of molecular motion, and can overcome the effects of radicals and charges in order to form Ps. Since the induced radical numbers and the charging effect should be similar in the irradiated and non-irradiated samples, the decrease in $I_{3}$ 
at the beginning of sample irradiation may be attributed to a smaller number of trapped electrons. This is due to the capture of ionized electrons by radicals being induced in the irradiated samples by $\gamma$-irradiation before a PALS measurement. Hence, if radicals are the main effect to Ps formation, $I_{3}$ cannot increase, and is also expected to be constant. However, $I_{3}$ decreases with the elapsed time of the PALS measurement. In order to explain this decrease, another effect, such as an electric field created by positive charges, must be introduced. Positive charges are constantly injected into samples from positron sources; also, through the capture of ionized electrons by radicals positive charges accumulate faster than charging from the sources.

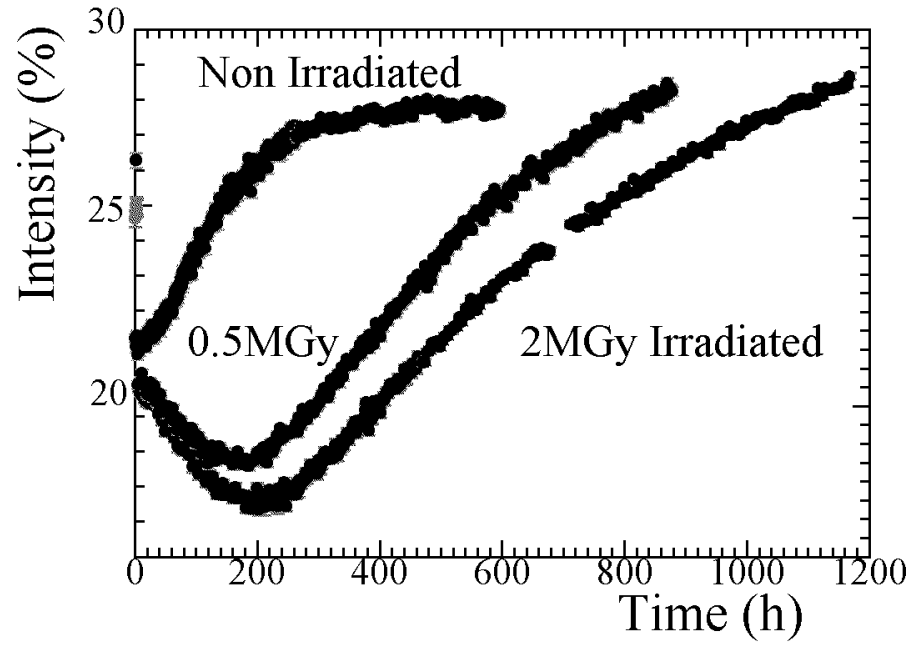

Fig. 5. o-Ps intensities versus elapsed time at the liquid nitrogen temperature for non-irrradiated, 0.5 MGy irradiated, and 2 MGy irradiated PC samples.

The charging effect has been considered as one of effects to reduce Ps formation $[11,19]$. In our study, the effect can be estimated as follows. A positron source of $3.7 \mathrm{MBq}$ emits half of the source activities $\left(1.85 \mathrm{Me}^{+} / \mathrm{s}\right)$ towards one side of the samples. Half of these positrons are accumulated within a region covered by $\mathrm{e}^{+}$penetration - source diameter $(2 \mathrm{~mm}) \times$ depth $(\approx 30 \mu \mathrm{m})$. Then the positron density can be estimated to be $\approx 3.5 \times 10^{19} \mathrm{e}^{+} /\left(\mathrm{h} \cdot \mathrm{m}^{3}\right)$ from the $3.7 \mathrm{MBq}$ strong source. If these positrons are distributed uniformly, the distance between two charges is roughly $30 \mathrm{~nm}$. If injected positrons locate $15 \mathrm{~nm}$ away from one positive charge, an electric field of $64 \mathrm{kV} / \mathrm{cm}$ is expected from one charge. This suggests that, since the electric field may become larger than $30 \mathrm{kV} / \mathrm{cm}$, which was measured to affect Ps formation in nonpolar liquids [11], there is a large possibility to affect Ps formation.

\section{Conclusion}

In order to explain the change of $I_{3}$ during a PALS experiment, a charging effect due to positrons injected by the positron sources must be considered $[11,19]$ 
in addition to radicals, cross-linking, and a structure change. We observed that the decreasing rate of $I_{3}$ was the same for irradiated and non-irradiated samples in the PALS measurement using a strong source; also, during the beginning of the PALS measurement at the liquid temperature, $I_{3}$ decreased (increased) for irradiated (non-irradiated) samples. It seems that radicals, cross-linking, and structure changes cannot explain these changes of $I_{3}$.

\section{Acknowledgment}

This work was supported by a Grant-in-Aid in Scientific Research by the Japanese Ministry of Education, Culture, Sports, and Science. We are indebted to the Inter-University Laboratory for the Common Use of JAERI Facilities for ${ }^{60} \mathrm{Co}$ $\gamma$-ray irradiation.

\section{References}

[1] P. Kindl, G. Reiter, Phys. Status Solidi A 104, 707 (1987).

[2] B. Levay, M. Lalovic, H.J. Ache, J. Chem. Phys. 90, 3282 (1989).

[3] T. Suzuki, T. Miura, Y. Oki, M. Numajiri, K. Kondo, Y. Ito, J. Phys. IV, Coll. C4, suppl. au J. Phys. II (France) 3, 283 (1993).

[4] A. Uedono, T. Kawano, S. Tanigawa, M. Ban, M. Kyoto, T. Uozumi, J. Polym. Sci. B 35, 1601 (1997).

[5] A.J. Hill, P.J. Jones, P.J. Lind, G.W. Pearsall, J. Polym. Sci. A 26, 1541 (1988).

[6] M. Walander, F.H.J. Maurer, Mater. Sci. Forum 105-110, 1811 (1992).

[7] T. Suzuki, T. Miura, Y. Oki, M. Numajiri, K. Kondo, Y. Ito, Radiat. Phys. Chem. 45, 657 (1995).

[8] T. Hirade, C.L. Wang, F.H. Maurer, M. Eldrup, N.J. Pederson, in: The 35th Annual Meeting on Radioisotopes in the Physical Science and Industries, Tokyo 1998, Book of Abstracts, 1998, p. 89.

[9] T. Hirade, F.H. Maurer, M. Eldrup, Radiat. Phys. Chem. 58, 465 (2000).

[10] Y. Ito, T. Hirade, E. Hamada, T. Suzuki, Y. Ito, Acta Phys. Pol. A 95, 533 (1999).

[11] Y. Ito, in: Positron and Positronium Chemistry, Eds. D.M. Schrader, Y.C. Jean, Elsevier, Amsterdam 1988, Ch. 4, p. 120.

[12] A. Badia, G. Duplatre, Radiat. Phys. Chem. 54, 151 (1999).

[13] T. Hirade, T. Kumada, to be published in Radiat. Phys. Chem.

[14] T. Suzuki, Yuraka Ito, K. Kondo, E. Hamada, Yasuo Ito, Radiat. Phys. Chem. 58, $485(2000)$.

[15] P. Kirkegaard, M. Eldrup, O.E. Mogensen, N. Pedersen, PATFIT 88, Ris $\emptyset-M-2740$, Ris $\varnothing$ National Lab., Roskilde (Denmark) 1989.

[16] J.H. Golden, E.A. Hazell, J. Polym. Sci. 1, 1671 (1963).

[17] J.H. Golden, B.L. Hammant, E.A. Hazell, J. Polym. Sci. 2, 4787 (1964).

[18] T. Suzuki, Y. Namito, T. Miura, Y. Oki, M. Numajiri, H. Hirayama, K. Kondo, Y. Ito, KEK Preprint 94-121 (1994).

[19] P.G. Coleman, S. Kuna, R. Grynszpan, Mater. Sci. Forum 255-257, 668 (1997). 